\title{
Micro-Sensors for Space Applications
}

\author{
PRECEMPG
}

JAN 242000

\author{
M. A. Butler, G. C. Frye-Mason, and G. C. Osbourn \\ Microsensor R \& D Department, 1715 \\ Sandia National Laboratories \\ Albuquerque, NM 87185-1425
}

\begin{abstract}
Important factors in the application of sensing technology to space applications are low mass, small size, and low power. All of these attributes are enabled by the application of MEMS and micro-fabrication technology to microsensors. Two types of sensors are utilized in space applications: remotes sensing from orbit around the earth or another planetary body, and point sensing in the spacecraft or external to it. Several Sandia projects that apply microfabrication technologies to the development of new sensing capabilities having the potential for space applications will be briefly described. The Micro-Navigator is a project to develop a MEMS-based device to measure acceleration and rotation in all three axes for local area navigation. The Polychromator project is a joint project with Honeywell and MIT to develop an electrically programmable diffraction grating that can be programmed to synthesize the spectra of molecules. This grating will be used as the reference cell in a gas correlation radiometer to enable remote chemical detection of most chemical species. Another area of research where microfabrication is having a large impact is the development of a "lab on a chip." Sandia's efforts to develop the $\mu$ ChemLab ${ }^{\mathrm{TM}}$ will be described including the development of microfabricated pre-concentrators, chromatographic columns, and detectors. Smart sensors that allow the spacecraft independent decision making capabilities depend on pattern recognition. Sandia's development of a new pattern recognition methodology that can be used to interpret sensor response as well as for target recognition applications will be described.
\end{abstract}




\section{DISCLAIMER}

This report was prepared as an account of work sponsored by an agency of the United States Government. Neither the United States Government nor any agency thereof, nor any of their employees, make any warranty, express or implied, or assumes any legal liability or responsibility for the accuracy, completeness, or usefulness of any information, apparatus, product, or process disclosed, or represents that its use would not infringe privately owned rights. Reference herein to any specific commercial product, process, or service by trade name, trademark, manufacturer, or otherwise does not necessarily constitute or imply its endorsement, recommendation, or favoring by the United States Government or any agency thereof. The views and opinions of authors expressed herein do not necessarily state or reflect those of the United States Government or any agency thereof. 


\section{DISCLAIMER}

Portions of this document may be illegible in electronic image products. Images are produced from the best available original document. 


\section{INTRODUCTION}

Utilization of sensing technology in space related applications, imposes additional constraints on the technology in terms of size, weight, and power requirements. These requirements to minimize space utilization and power consumption are met nicely by the application of MEMS and micro-fabrication methods to the fabrication of sensing devices. Two kinds of sensing applications can be envisioned in space, remote sensing from the spacecraft of a planetary, asteroid or comet surface and point sensing inside or outside of the spacecraft. Here we will present several technologies being developed at Sandia National Laboratories that can impact sensing applications in space.

\section{MICRO-NAVIGATOR}

The creation of microsystems (e.g., that sense, think, act, or communicate) often requires electronic circuitry coupled with mechanical elements. The monolithic integration of electronic circuitry on the same chip as electro-mechanical devices has many advantages over approaches that involve complex multi-chip, packaging schemes. Batch fabrication of "systems on a chip" enables very low cost production. By reducing the number of components in the system, significantly improved system reliability may be achieved. For example, reducing the chip count, eliminating the bond wires connecting electrical to mechanical circuits, and reducing the complexity of the packaging/assembly process all benefit reliability. Finally, monolithic integration enables overall system performance, particularly for microsensing systems, to be increased by many orders of magnitude by reducing electrical interconnect parasitics, such as capacitance.

A long-term goal of a micromachined micro-navigation system requires both 3-axis accelerometers and gyroscopes. Multi-axis, vibratory-rate gyros have been fabricated with this technology and they are undergoing testing at this time. These devices are manufactured on the same silicon substrate as the 3-axis accelerometer and they will form the heart of a future micro-navigation system. With this incredible, enabling technology, one can imagine a vast number of new applications from low-cost automotive safety systems to micro-satellite navigation systems.

\section{POLYCHROMATOR}

An algorithm has been developed that calculates diffraction grating profiles that diffract multiple wavelengths at the same angle [1]. This development extends the application of diffractive optics from spatial domain optical processing into the spectral domain. This algorithm can be used to define novel diffraction gratings that are complex spectral filters with any spectral content desired. In fact, they can even be used to synthesize the absorption spectra of specific molecules [2]! Gratings have been successfully fabricated in silicon using conventional lithographic methods for $\mathrm{HF}$, toluene, methyl isobutyl ketone, and kerosene and the diffracted beam 
successfully reproduces the spectra of these molecules. One space related application of this technology would be to replace the reference cell in a correlation radiometer for remote optical sensing. These gratings offer the ability to use correlation radiometry with transient or dangerous chemical species, and to greatly reduce the size of the reference cell for weak absorption lines. The gratings can also reproduce the complement of the transmission spectrum for a specific molecule in the reference cell rather than the transmission spectrum. This greatly reduces the unmodulated light background and thus the noise level in the detector. A joint project is underway with Honeywell and MIT as partners to develop a programmable grating structure [3] that will allow real-time reconfiguration of the grating elements to produce any spectral band-pass filter one desires. This capability allows spectral modulation of the diffracted beam in such a way as to enhance the ability of the radiometer to distinguish between chemical species with similar spectral features.

\section{$\mu$ ChemLab $^{\mathrm{TM}}$}

A miniature, integrated chemical laboratory $\left(\mu \mathrm{ChemLab}{ }^{\mathrm{TM}}\right)$ is being developed that utilizes microfabrication to provide faster response, smaller size, and an ability to utilize multiple analysis channels for enhanced versatility and chemical discrimination [4]. Our $\mu$ ChemLab ${ }^{\mathrm{TM}}$ program goal is to develop a small (palm-top computer sized), lightweight, and autonomous systems that provide rapid (1 min), sensitive (1-10 ppb), and selective detection of target analytes. Improved sensitivity and selectivity are achieved by using a cascaded approach where each channel includes a sample collector/concentrator, a gas chromatographic (GC) separator, and a chemically selective surface acoustic wave (SAW) array detector. Prototypes of all three components have been developed and demonstrated and current work is focused on integrating these into a complete analysis system.

The sample collector/pre-concentrator requires a medium to absorb the vapor sample to be analyzed and a method to desorb the accumulated sample in a short pulse. Microfabrication methodology has been used to fabricate "micro-hotplates" [5] that provide the means of rapidly heating a surface with limited expenditure of energy. Thus, the micro-hotplate structure can be utilized as a sample collector with the addition of a coating to absorb and concentrate the sample. This is the structure chosen for the $\mu$ ChemLab ${ }^{\mathrm{TM}}$.

Gas chromatographic (GC) separation, used to separate compounds in time, is a powerful tool for enhancing chemical discrimination. While conventional GC systems utilize micro-capillary columns, miniature on-chip GC columns can provide improved ruggedness, smaller size, lower power, and an ability to integrate other components such as pre-concentrators and detectors. We have developed a novel fabrication technique for making columns up to one meter long on a one- $\mathrm{cm}^{2}$ chip. These columns are fabricated using high aspect ratio silicon etching (HARSE) $[5,6]$ to form a high aspect ratio column that is narrow and deep (typical columns 40-80 $\mu \mathrm{m}$ wide by $>350 \mu \mathrm{m}$ deep). This reactive ion etching process results in parallel sidewalls providing a constant column width, a critical feature to achieve effective 
separations. With only 5 psi of pressure (miniature diaphragm pumps can provide this quite easily), we have demonstrated separation of up to five components in less than 30 seconds.

To enable selective detection, miniature SAW sensor arrays containing four 380 $\mathrm{MHz}$ SAWs with an active coated area of less than one $\mathrm{mm}^{2}$ have been developed [7]. In addition, an integrated sensor measuring about $0.2 \mathrm{~cm}^{2}$ containing the SAW array and all high frequency electronics has been designed. This device is being fabricated using GaAs, a unique substrate since it is piezoelectric and is an excellent semiconductor for fabricating high frequency electronics. This design incorporates amplifiers to provide the gain and phase comparators to provide a DC output proportional to SAW phase. All components have been demonstrated in discrete versions and the integrated design should provide $\mathrm{DC}$ in/DC out operation.

The $\mu$ ChemLab ${ }^{\mathrm{TM}}$ can provide chemical analysis capability in a small package for use on a planetary surface or inside a manned spacecraft for analysis of the cabin atmosphere or biomedical evaluation of the cabin occupants. The micro-components developed for this device can also be combined in more complex structures in order to solve other chemical and biochemical monitoring problems.

\section{VERI PATTERN RECOGNITION METHODOLOGY}

Interpretation of the response of an array of chemical sensors is handled very successfully by a new pattern recognition methodology developed at Sandia. This non-parametric pattern recognition (PR) method is based on the visual empirical region of influence (VERI) clustering technique [10] invented at Sandia. This method is based on an evaluation and modeling of cluster analysis performed visually by human beings in two dimensions. The resulting algorithm has been generalized to $n$ dimensions and can be applied to the evaluation of data sets ranging from sensor arrays to visual images. The VERI PR algorithm provides a powerful, general-purpose alternative both to statistical analysis methods and popular nonparametric methods (e.g. neural nets and k-nearest neighbors). VERI evidently provides the first effective pattern recognition technique that is completely automated, i.e. problem-dependent training optimization and outlier thresholds are not provided by the user. The VERI method implicitly contains multivariate "goodness-of-matching" thresholds, derived from human clustering threshold judgments, which are functions of the arbitrarily complex multivariate shape of each class in the data. This provides fully automated performance on arbitrarily complex data distributions, without risk of "over-learning", that can exceed the best results achievable with even careful optimization of neural net or k-nearest-neighbor approaches. The method automatically recognizes atypical outliers and automatically detects data for which multiple class matches are acceptable. This is accomplished in a truly multivariate fashion, rather than with the simple univariate reject thresholds that are commonly adjusted in other approaches. These properties are especially useful for pattern recognition in uncontrolled/ unpredictable environments and for suppressing false alarms. VERI also provides an efficient, 
automated approach for directly searching for optimal feature sets for pattern recognition and data mining applications. The automated VERI approach allows an inexperienced user to correctly apply PR to problems in which unknown classes or incompletely distinguishable classes may be encountered, without the need to supply any additional information. Further information about application of the VERI method to sensor arrays is available at Sandia's web site [11].

\section{SUMMARY}

As illustrated by the above examples, the general trend in micro-sensor and micro-instrument research and development is to small size and lower power devices. Both of these trends increase the potential for utilization of these new technologies to applications in space. The miniaturization trend will impact both remote sensing and point sensing applications since the key issue is launch mass of the spacecraft.

\section{ACKNOWLEDGEMENTS}

Sandia is a multi-program laboratory operated by Sandia Corporation, a Lockheed Martin Company, for the United States Department of Energy under Contract DEAC04-94AL85000. 


\section{REFERENCES}

1. "Synthetic Spectra: a Tool for Correlation Spectroscopy," M. B. Sinclair, M. A. Butler, A. J. Ricco, and S. D. Senturia, Applied Optics, 36, 3342 (1997).

2. "Synthetic Infrared Spectra," M. B. Sinclair, M. A. Butler, S. H. Kravitz, W. J. Zubrzycki, and A. J. Ricco, Optics Letters, 22, 1036 (1997).

3. "ELECTRICALLY PROGRAMMABLE DIFFRACTION GRATING" A. J. Ricco, M. A. Butler, M. B. Sinclair, and S. D. Senturia, US patent \# 5,757,536 issued May 26, 1998.

4. "Integrated Chemical Analysis Systems for Gas Phase CW Agent Detection," G. C. Frye-Mason, R. J. Kottenstette, E. J. Heller, C. M. Matzke, S. A. Casalnuovo, P. R. Lewis, R. P. Manginell, W. K. Schubert, V. M. Hietala, and R. J. Shul, Proc. $\mu$ TAS '98 Workshop, Kluwer Academic Publishers, Boston, pp. 477-480 (1998).

5. "Microfabrication Of Membrane-Based Devices by HARSE and Combined HARSE/Wet Etching", R. P. Manginell, G. C. Frye, R. J. Shul and C. G. Willison, Proc. SPIE Micromachining and Microfabrication Conference, Vol. 3511, pp. 269-276, (1998).

6. "Microfabricated Silicon Gas Chromatographic Micro-Channels: Fabrication and Performance," C. M. Matzke, R. J. Kottenstette, S. A. Casalnuovo, G. C. FryeMason, M. L. Hudson, D. Y. Sasaki, R. P. Manginell, and C. C. Wong. Proc. SPIE Micromachining and Microfabrication Conference, Vol. 3511, pp. 262-268 (1998).

7. "Acoustic Wave Chemical Microsensors in GaAs," S. A. Casalnuovo, E. J. Heller, V. M. Heitala, A. G. Baca, R. Kottenstette, S. L. Heitala, J. L. Reno, and G. C. Frye-Mason, Proc. SPIE Micromachining and Microfabrication Conference, Vol. 3511, pp. 103-110 (1998).

8. "Empirically defined regions of influence for clustering analyses," G. C. Osbourn and R. F. Martinez, Pattern Recognition 28, 1793 (1995).

9. "A New Approach to Sensor Array Analysis Applied to Volatile Organic Compound Detection: Visual Empirical Region of Influence (VERI) Pattern Recognition," G. C. Osbourn, J. W. Bartholomew, A. J. Ricco, and G. C. Frye (http://www.sandia.gov/1100/1155Web/chempap.htm). 